\title{
Microbiological Profile of Neonatal Sepsis at a Maternity Hospital in Omdurman, Sudan
}

Corresponding Author: Manal Abdelaziz;

email:

manalhassieb@gmail.com

Received 10 February 2019

Accepted 26 March 2019

Published 31 March 2019

Production and Hosting by

Knowledge E

(c) Manal Abdelaziz et al. This article is distributed under the terms of the Creative

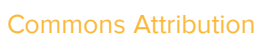

which permits

unrestricted use and

redistribution provided that the original author and source are credited.

Editor-in-Chief:

Prof. Mohammad A. M. Ibnouf

\section{Manal Abdelaziz¹, Yassir Hamadalnil ${ }^{1}$, Omaima Hashim² ${ }^{2}$ Tahane Bashir ${ }^{3}$, and E. S. Mahjoub ${ }^{4}$}

${ }^{1}$ Department of Microbiology, Maternity Hospital, Khartoum

${ }^{2}$ Department of Microbiology and Virology, National Center for Gastrointestinal \& Liver Diseases, Khartoum

${ }^{3}$ Department of Microbiology, Radioisotope Center Khartoum, Sudan

${ }^{4}$ Department of Microbiology, Red Sea University, Port Sudan

${ }^{5}$ Department of Microbiology, University of Khartoum, Khartoum, Sudan

\section{Abstract}

Background: Neonatal sepsis is a clinical syndrome characterized by systemic signs of infection and accompanied by bacteremia in the first month of life. It is a major cause of morbidity and mortality in neonatal period. The study was conducted to determine microbiological profile and antibiogram of neonatal sepsis at Omdurman Maternity Hospital.

Methods: This was a cross-sectional hospital-based study involving 202 neonatal blood cultures at Omdurman Maternity Hospital during the period from April 2017 to April 2018. Specimens were cultured in Brain Heart Infusion broth followed by subculture of isolates on blood agar, MacConkey agar, and Chocolate agar and incubated aerobically at $37^{\circ} \mathrm{C}$ for $24 \mathrm{~h}$. The isolates were tested for their susceptibility to antimicrobial agents using the Kirby Bauer disc diffusion method.

Results: Of 202 positive blood cultures, 130 cases (64.4\%) were early onset and 72 cases (35.6\%) were recorded for late onset sepsis. Gram-negative pathogens approaching $(123,60.9 \%)$. Staphylococcus aureus was the most common organism in both groups of neonatal sepsis being isolated from (71, 35.7\%), followed by Klebsiella pneumoniae (43, 21.2\%). Gram-negative organisms were sensitive to Imepenem (97.3\%) and Meropenem (80.5\%) and resistant to third-generation Cephalosporins (65.3\%) and Amoxicillin/Clavulanic acid (91.4\%). Gram-positive organisms were resistant to Cefotaxime (75\%), Amoxicillin/Clavulanic acid (65.4\%), and Clindamycin (68.2\%); 91.6\% of gram-positive isolates were sensitive to Vancomycin.

Conclusion: Gram-negative pathogens took the major spectrum of isolates. Klebsiella pneumoniae (21.2\%) was the most frequent gram-negative organism. Methicillinresistant Staphylococcus aureus (MRSA) (33.7\%) was the most common isolate. Most of the isolates were multidrug resistant. The best choice for treatment is Vancomycin (8.4\%) and Imepenem (2.7\%) for gram-positive and gram-negative, respectively. Adherence to antibiotic policy, antimicrobial surveillance, and policy updating is necessary.

Keywords: Neonatal sepsis, MRSA, ESBL 


\section{Introduction}

Neonatal sepsis is considered one of the major causes of morbidity and mortality among term and preterm infants in neonatal intensive care units (NICU) [1]. Neonatal sepsis is a systemic infection occurring in infants less than 28 days of life and is classified into early onset and late onset neonatal sepsis. Early-onset neonatal sepsis (EOS) occurs within seven days of birth, while late-onset neonatal sepsis (LOS) occurs after the first seven days of life [2]. Neonatal infections are responsible for about 1.6 million deaths every year in developing countries [3]. Sepsis and meningitis are considered the most common causes of these deaths [4]. In developed countries, Streptococcus agalactiae (GBS) and Escherichia coli were the most common pathogens isolated in severe cases [5]. EOS is caused by GBS, E. coli, Streptococcus viridans, Staphylococcus aureus, and Haemophilusinfluenzae [6]. The infant may acquire the pathogen either in utero or intrapartum [7]. According to the World Health Organization (WHO), there were around four million neonatal deaths every year, about a quarter of them is due to neonatal sepsis/pneumonia alone [8]. In Sudan, the prevalence of neonatal sepsis is $17.5 \%$ and the mortality is $14.5 \%$ [9]. This study was conducted to determine the microbiological profile and antibiogram of neonatal sepsis at Omdurman Maternity Hospital.

\section{Materials and Methods}

The present study was a cross sectional, hospital-based study, conducted at Omdurman Maternity Hospital during the period April 2017-April 2018. In this period, a total of 202 positive blood samples were analyzed. Two $\mathrm{ml}$ of blood from neonate was dispensed into a sterilized universal bottle containing brain heart infusion broth to make a 1:10 dilution. It was collected from neonates under aseptic condition. Each sample was cultured in blood culture bottle and incubated aerobically at 37c up to seven days. Regular observation of the bottle color, and turbidity, direct gram stain, and subculture were done to detect the positive ones. Regular subculture was done after one day of incubation, at 48 hours, and then at 96 hours. Each sample was subcultured on blood agar, MacConkey agar, and Chocolate blood agar and incubated aerobically at $37^{\circ} \mathrm{C}$ for $24 \mathrm{~h}$. If there was no broth change or negative subculture for up to seven days, blood culture was considered as negative. Identification was done based on colonial morphology, gram stain, and standard biochemical tests. Antimicrobial susceptibility of isolated organisms was detected by using the Kirby Bauer disc diffusion method according to the Clinical and Laboratory Standard Institute (CLSI) [10]. Pure colonies 
of isolates were emulsified in distilled water and inoculated on Muller Hinton agar. Antimicrobial discs were placed on the inoculated agar and incubated for $24 \mathrm{~h}$ at $37^{\circ} \mathrm{C}$; then they were observed for zones of inhibition and determined as susceptible, intermediate, or resistant according to the CLSI [10]. The applied antibiotic for grampositive cocci included oxacillin to check MRSA and for gram-negative rods $E$. coli ATTCC 25922 was used as control strain. Data were analyzed by statistical package for social science SPSS software version 24 . Chi-square tests were done. $P$-value $<0.05$ was considered significant in comparative data.

\section{Results}

A total of 202 newborn babies were enrolled in this study. There were 130 cases (64.4\%) of early-onset sepsis and 72 cases (35.6\%) of late-onset sepsis, see Table 1. Among the positive blood cultures, gram-negative septicemias were 123(60.9\%), while gram-positive were 75(37.1\%). Staphylococcus aureus was isolated from 71(35.7\%) in both groups of neonatal sepsis, followed by Klebsiella pneumoniae 43(21.2\%) Table 2. Tables 3 and 4 show the result of antibiotics sensitivity tests.

TABLE 1: Distribution of isolated organisms in an EOS and LOS.

\begin{tabular}{l|l} 
& \\
Bacteria & Streptococcus pyogenes \\
& Klebsiella oxytoca \\
& Candida albicans \\
& Enterococcus faecalis \\
& Proteus mirabilis \\
& Salmonella spp. \\
& S. epidermidis \\
\hline Enterobacter spp. \\
Acinetobacter baumannii \\
\hline Serratia marcescens \\
Citrobacter spp. \\
\hline Pseudomonas aeruginosa \\
\hline MRSA \\
\hline Staphylococcus aureus \\
Klebsiella ESBL \\
\hline Klebsiella pneumonae \\
E.coli ESBL \\
\hline E. coli \\
\hline
\end{tabular}

\begin{tabular}{|c|c|c|}
\hline $\begin{array}{c}\text { Frequency } \\
\text { of Isolation }\end{array}$ & Early Onset & Late Onset \\
\hline 1 & 1 & 0 \\
\hline 1 & 1 & 0 \\
\hline 4 & 0 & 4 \\
\hline 2 & 0 & 2 \\
\hline 1 & 1 & 0 \\
\hline 6 & 3 & 3 \\
\hline 1 & 0 & 1 \\
\hline 6 & 5 & 1 \\
\hline 5 & 2 & 3 \\
\hline 7 & 7 & 0 \\
\hline 4 & 1 & 3 \\
\hline 18 & 12 & 6 \\
\hline 68 & 43 & 25 \\
\hline 3 & 2 & 1 \\
\hline 11 & 8 & 3 \\
\hline 32 & 19 & 13 \\
\hline 9 & 7 & 2 \\
\hline 23 & 18 & 5 \\
\hline 202 & 130 & 72 \\
\hline & & \\
\hline 1 & & 0 \\
\hline
\end{tabular}


TABLE 2: Frequencies of bacteria that cause neonatal sepsis in which MRSA is the most common organism $(n=202)$.

\begin{tabular}{|l|l|c|c|}
\hline & & Frequency & Percent \\
\hline $\begin{array}{l}\text { Type of } \\
\text { Bacteria }\end{array}$ & MRSA & 68 & 33.7 \\
\hline & Klebsiella pneumonae & 32 & 15.8 \\
\hline E. coli & 23 & 11.4 \\
\hline Pseudomonas aeruginosa & 18 & 8.9 \\
\hline Klebsiella ESBL & 11 & 5.4 \\
\hline E. coli ESBL & 9 & 4.5 \\
\hline Serratia marcescens & 7 & 3.5 \\
\hline Enterobacter spp. & 6 & 3.0 \\
\hline Salmonella spp. & 6 & 3.0 \\
\hline Acinetobacter baumannii & 5 & 2.5 \\
\hline Citrobacter spp. & 4 & 2.0 \\
\hline Candida albicans & 4 & 2.0 \\
\hline Staphylococcus aureus & 3 & 1.5 \\
\hline Enterococcus faecalis & 2 & 1.0 \\
\hline S. epidermidis & 1 & .5 \\
\hline Proteus mirabilis & 1 & .5 \\
\hline Klebsiella oxytoca & 1 & .5 \\
\hline Streptococcus pyogenes & 100.0 \\
\hline
\end{tabular}

TABLE 3: Frequencies of antibiotic susceptibility of the gram-negative organisms.

Antibiotics
Imipenem
Meropenem
Cefotaxime
Amikacin
Ceftazidime
Gentamicin
Amoxicillin/clavulanic acid
Penicillin

\begin{tabular}{|c|}
\hline Sensitive \\
\hline $110(97.3 \%)$ \\
\hline $95(80.5 \%)$ \\
\hline $43(40 \%)$ \\
\hline $45(46.9 \%)$ \\
\hline $36(34.7)$ \\
\hline $32(39.5 \%)$ \\
\hline $6(8.6 \%)$ \\
\hline $7(13.5 \%)$ \\
\hline
\end{tabular}

\begin{tabular}{|c|}
\hline Resistant \\
\hline $3(2.7 \%)$ \\
\hline $23(19.5 \%)$ \\
\hline $66(60 \%)$ \\
\hline $51(53.1 \%)$ \\
\hline $68(65.3 \%)$ \\
\hline $49(60.5 \%)$ \\
\hline $64(91.4 \%)$ \\
\hline $45(86.5 \%)$ \\
\hline
\end{tabular}

\section{Discussion}

Neonatal sepsis can be defined as a clinical syndrome characterized by systemic signs of infection and accompanied by bacteremia in the first month of life [11]. It is considered one of the major causes in neonatal morbidity and mortality [1]. The pathogens causing neonatal sepsis in developing countries differ from those in developed countries [12]. But overall the gram-negative organisms are more common [13]. 
TABLE 4: Frequencies of antibiotic susceptibility of the gram-positive organisms.

\begin{tabular}{|l|c|c|}
\hline Antibiotics & Sensitive & Resistant \\
\hline Penicillin & $6(9 \%)$ & $61(91 \%)$ \\
\hline Vancomycin & $66(91.6 \%)$ & $6(8.4 \%)$ \\
\hline Amoxicillin/Clavulanic acid & $12(34.6 \%)$ & $40(65.4 \%)$ \\
\hline Ciprofloxacin & $26(43.3 \%)$ & $34(56.7 \%)$ \\
\hline Cefotaxime & $2(25 \%)$ & $6(75 \%)$ \\
\hline Clindamycin & $7(31.8 \%)$ & $15(68.2 \%)$ \\
\hline Gentamicin & $45(60.8 \%)$ & $29(39.2 \%)$ \\
\hline
\end{tabular}

In this study, gram-negative and gram-positive organisms encountered $60.9 \%$ and $37.1 \%$, respectively, which is comparable to a study conducted in India [12], which reported that gram-negative and gram-positive organisms were $56 \%$ and $44 \%$, respectively. Similar results were reported in Iraq [14] and Tanzania [15].

In this study, the EOS and LOS were $64.6 \%$ and $35.4 \%$, respectively. This result is also comparable to a study conducted in India [16], which reported that EOS and LOS were $61.41 \%$ and $38.59 \%$, respectively, further supported by another study done in India [17]. However, it differs from the study conducted in Tanzania [15] and Iraq [14]. Staphylococcus aureus was the most common isolate (35.1\%) in both EOS and LOS in the current study, and 95\% of S. aureus were Methicillin-resistant Staphylococcus aureus (MRSA). This result is similar to a study conducted by Zaidi et al. [18]. The second most common isolate was Klebsiella pneumoniae (21.2\%), from which $25.58 \%$ were Extended Spectrum Beta Lactamase (ESBL). High resistance rate to cephalosporins can be contributed to the irrational use of these antibiotics. The presence of Proteus mirabilis, Serratia marcescens, Klebsiella oxytoca, and Streptococcus pyogenes isolates in the EOS and the absence of these isolates in the LOS was noted in this study.

Antimicrobial resistance now is worldly distributed and multidrug-resistant organisms are a global concern. Antibiotics susceptibility patterns differ from one country to another, so it is difficult to compare the susceptibility patterns between countries. In this study, gram-negative organisms showed a high sensitivity to Imepenem (97.3\%) which is consistent to the local study conducted at Soba Hospital in Khartoum (100\%) [19]. Imepenem sensitivity was $80.5 \%$. This study reflected moderate resistance to Amikacin (53.1\%) and Gentamicin (60.5\%), which differs from the local study that showed low resistance to Amikacin (10.5\%) and high resistance to Gentamicin (89.5\%) [19]. This study reflected a moderate resistance to third generation cephalosporin $60 \%$ when it's compared to the local study that reflected high resistance (89.5\%) [19]. The best sensitivity of gram-positive organisms was to Vancomycin (91.6\%), most of these isolates 
were MRSA. This result was comparable to Soba study (92\%) [19]. Gentamicin showed moderate sensitivity (60\%), which is also comparable to the Soba study (50\%) [19]. Also, for the gram-positive organisms, the third generation cephalosporin showed a moderate resistance (75\%). Most probably the overuse of third-generation cephalosporin is a main cause of the wide range resistance.

\section{Conclusion}

From this study, gram-negative pathogens took a major spectrum of isolates. Klebsiella pneumoniae was the most frequent gram-negative organism. Methicillin-resistant Staphylococcus aureus (MRSA) was the most common isolate. Most isolates were multidrug resistance. Therefore, the surveillance for antimicrobial resistance was necessary. Adhering to and updating the antibiotic policy contributes to containing this problem.

\section{References}

[1] Camacho-Gonzalez, A., Spearman, P. W., and Stoll, B. J. (2013). Neonatal infectious diseases: evaluation of neonatal sepsis. Pediatric Clinics of North America, vol. 60, pp. 367-389.

[2] Verani, J. R., McGee, L., Schrag S. J. (November 19, 2010). Prevention of perinatal group B streptococcal disease: revised guidelines from CDC. MMWR Recommendations and Reports, vol. 59, pp. 1-32.

[3] Brye, J, Boschi, Pinto, C., et al. (2005). WHO estimate of the causes of death in children. Lancet, vol. 365, p. 1147.

[4] NBS, S., Menezes, R. P., Brito, M. O., et al. (2017). Sepsis neonatal: epidemiology, etiology and risk factors. Advances in Biotechnology and Microbiology, vol. 4, no. 2, pp. 7-8.

[5] Le Doare, K. and Heath, P. T. (2013). An overview of global GBS epidemiology. Vaccine, vol. 31S, pp. D7-12.2.

[6] Weston, E. J., Pondo, T., Lewis, M. M., et al. (November 2011). The burden of invasive early-onset neonatal sepsis in the United States, 2005-2008. The Pediatric Infectious Disease Journal, vol. 30, no. 11, pp. 937-941.

[7] Simonsen, K. A., Anderson-Berry, A. L., Delair, S. F., et al. (January 1, 2014). Earlyonset neonatal sepsis. Clinical Microbiology Reviews, vol. 27, no. 1, pp. 21-47.

[8] World Health Organiation. (2011). Neonatal Sepsis - A Major Killer to be Tackled in Communities. WHO. 
[9] Kheir, A. E. M. and Khair, R. A. (2014). Neonatal sepsis: Prevalence and outcome in a tertiary neonatal unit in Sudan. Time Journals of Medical Sciences Report and Research, vol. 2, no. 1, pp. 21-25.

[10] Clinical \& Laboratory Standards Institute. (2011). Performance Standards for Antimicrobial Susceptibility Testing: Twenty-First Informational Supplement. CLSI document M100-S21. Wayne, PA: Clinical and Laboratory Standards Institute.

[11] Verma, P., Berwal, P. K., Nagaraj, N., et al. (2015). Neonatal sepsis: Epidemiology, clinical spectrum, recent antimicrobial agents and their antibiotic susceptibility pattern. International Journal of Contemporary Padiatrics, vol. 2, no. 5, pp. 176180.

[12] Jyothi, P., Basavaraj, M. C., and Basavaraj, P. V. (2013). Bacteriological profile of neonatal septicemia and antibiotic susceptibility pattern of the isolates. Journal of Natural Science, Biology and Medicine, vol. 4, no. 2, pp. 306-309.

[13] Vergnano, S., Sharland, M., Kazembe, P., et al. (2005). Neonatal sepsis: An international perspective. Archives of Disease in Childhood - Fetal and Neonatal Edition, vol. 90, pp. F220-F224.

[14] Ibrahim, S. A. and Rahma, S. (2012). Microbiological profile of neonatal septicemia. The Iraqi Postgraduate Medical Journal, vol. 11, no. 1.

[15] Kayange, N., Kamugisha, E., Mwizamholya, D. L., et al. (2010). Predictors of positive blood culture and deaths among neonates with suspected neonatal sepsis in a tertiary hospital, Mwanza- Tanzania. BMC Pediatrics, pp. 1471-2431.

[16] Lamba, M., Sharma, R., Sharma, D., et al. (2016). Bacteriological spectrum and antimicrobial susceptibility pattern of neonatal septicaemia in a tertiary care hospital of North India. Journal of Maternal-Fetal and Neonatal Medicine, vol. 29, pp. 39933998.

[17] Agnihotri, N., Kaistha, N., and Gupta, V. (December 2004). Antimicrobial susceptibility of isolates from neonatal septicemia. Japanese Journal of Infectious Diseases, vol. 57, pp. 273-275.

[18] Zaidi, A. K., Thaver, D., Ali, S. A., et al. (2009). Pathogens associated with sepsis in newborns and young infants in developing countries. The Pediatric Infectious Disease Journal, vol. 28, pp. s10-s18.

[19] Babiker, W., Ahmed, A., Babiker, T., et al. (2018). Prevalence and causes of neonatal sepsis in Soba University Hospital, Sudan. Medical Microbiology Reports, vol. 1, 2. 